\title{
Carboxymethylchitosan/Poly(amidoamine) Dendrimer Nanoparticles in Central Nervous Systems-Regenerative Medicine: Effects on Neuron/Glial Cell Viability and Internalization Efficiency
}

\author{
António J. Salgado, ${ }^{\mathrm{a}}$ Joaquim M. Oliveira, ${ }^{\mathrm{a}}$ Rogério P. Pirraco, \\ Vitor H. Pereira, Joana S. Fraga, Alexandra P. Marques, Nuno M. Neves, \\ João F. Mano, Rui L. Reis, ${ }^{*}$ Nuno Sousa
}

The applicability of CMCht/PAMAM dendrimer nanoparticles for CNS applications was investigated. AFM and TEM observations revealed that the nanoparticles possessed a nanosphere-like shape with a size from 22.0 to $30.7 \mathrm{~nm}$. The nanoparticles could be bound to fluorescent-probe FITC for tracing purposes. Post-natal hippocampal neurons and cortical glial cells were both able to internalize the FITC-labeled CMCht/PAMAM dendrimer nanoparticles with high efficiency. The percentage of positive cells internalizing the nanoparticles varied, reaching a peak after $48 \mathrm{~h}$ of incubation. Further experiments for periods up to $7 \mathrm{~d}$ revealed that the periodical addition of FITC-labelled CMCht/ PAMAM dendrimer nanoparticles was needed to maintain the overall percentage of cells internalizing them. Finally, it was also observed that cell viability was not significantly affected by the incubation of dendrimer nanoparticles.

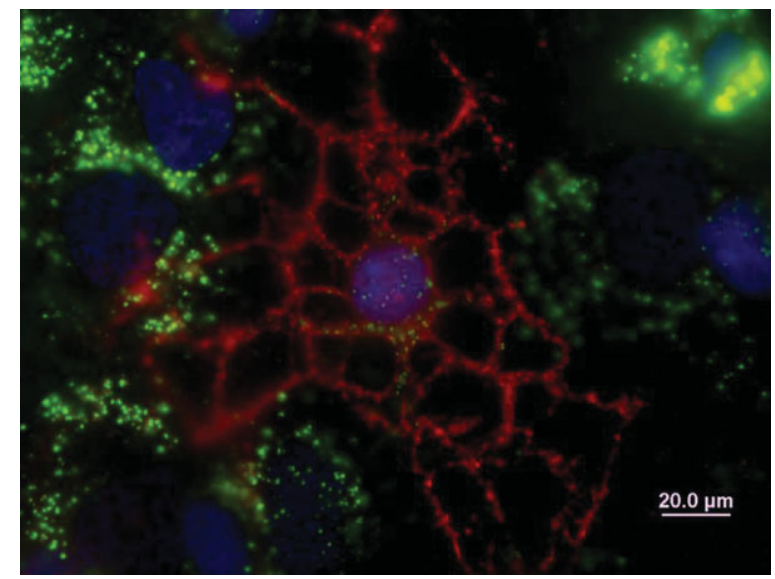

A. J. Salgado, V. H. Pereira, J. S. Fraga, N. Sousa

Life and Health Sciences Research Institute (ICVS), School of Health Sciences, University of Minho, 4710-057 Braga, Portugal J. M. Oliveira, R. P. Pirraco, A. P. Marques, N. M. Neves, J. F. Mano, R. L. Reis

3B's Research Group - Biomaterials, Biodegradables and Biomimetics, University of Minho, Headquarters of the European Institute of Excellence on Tissue Engineering and Regenerative Medicine, AvePark, 4806-909 Taipas, Guimarães, Portugal

Fax: +351 253510 90; E-mail: rgreis@dep.uminho.pt

J. M Oliveira, R. P. Pirraco, A. P. Marques, N. M. Neves, J. F. Mano,

R. L. Reis

IBB, Institute for Bioengineering and Biotechnology, PT Associated Lab, Braga, Portugal

${ }^{a}$ These authors contributed equally to the work

\section{Introduction}

Central nervous system (CNS) associated disorders, such as Parkinson's and Alzheimer's disease, multiple sclerosis, and spinal cord injury (SCI), are a leading cause of disability worldwide. In some of these disorders, namely in those involving large inflammatory processes such as SCI, it is common the administration of corticosteroids, as they are able to reduce inflammation. ${ }^{[1,2]}$ However, they also lead to drastic secondary effects, namely neuronal cell death and dendritic atrophy. ${ }^{[3]}$ Therefore, there is an urgent need for the development of new routes that can efficiently overcome this problem. 
One possible path to be followed could be through the use of nanomedicine-based approaches, namely those dealing with the development of nanoparticle-based targeted drugdelivery systems. ${ }^{[4-7]}$ The latter do not only possess the advantage of their size, but simultaneously can be surface engineered in order to present the adequate properties for biological interaction with the host cells/tissue, and thus deliver the drug to the desired cell population to be targeted. ${ }^{[4-7]}$ In general terms, drugs could be dissolved, entrapped, encapsulated or attached to a nanoparticle matrix, acting in this sense as a "Trojan horse" for a variety of chemical and biological entities. ${ }^{\left[{ }^{-10]}\right.}$ Moreover, as the nanoparticles can be internalized by CNS-derived cells, they are useful in the modulation of the activity of intracellular receptors such as those described for corticosteroids. ${ }^{[1]}$

For these reasons, several nanoparticle-based systems have been developed throughout the years. For instance, different authors have shown that polybutyl cyanoacrylate based nanoparticles can act as efficient dug delivery agents within the brain and CNS. ${ }^{[12-15]}$ Other studies ${ }^{[16-19]}$ have also revealed that liposomes, which commonly disclosed a non-toxic and biodegradable behavior, could be effective for the aforementioned applications. Other strategies have used polylactide-based nanoparticles derivatized with peptides for improved properties to cross the blood/brain barrier $^{[20,21]} \cdot(\mathrm{BBB})^{[20]}$ or poly(lactide-co-glycolide)-based nano-systems for the same purposes. ${ }^{[21]}$ Finally natural polymers, such as chitosan have also been used for nanoparticle drug delivery based approaches in CNSrelated applications. ${ }^{[22]}$ Still within this topic, dendrimers have also been put forward as yet another route for the proposed applications. ${ }^{[23-26]}$ Dendrimers are synthetic highly branched, spherical, monodisperse macromolecules of nanometer dimension. ${ }^{[23]}$ They are easily internalized by cells, a feature that might be a great advantage if the goal deals with, for instance, intracellular receptors of CNSderived cells. Their multiple surface functional groups can be easily modified to potentially attack a large number of ligands for a selected targeted drug delivery application, while its inner hydrophobic core is amenable for drug entrapment. ${ }^{[23,27,28]}$ However, the non-covalent encapsulation of drugs into dendrimers presents a general drawback in the sense that it is difficult to control the release of molecules from the dendrimer core. ${ }^{[28]}$ Furthermore, in order to increase the drug loading capacity, it is common to use higher generation of dendrimers (G7) with amine capping-groups, which have been shown to cause hemolysis and changes in red cell morphology, leading to a general overall cytotoxic behavior. ${ }^{[29]}$

In order to obviate these problems Oliveira et al. ${ }^{[30]}$ have recently proposed a new strategy consisting on a poly(amidoamine) (PAMAM) dendrimer core with grafted carboxymethylchitosan (CMCht) chains. This novel class of CMCht/PAMAM dendrimer nanoparticles could display a higher loading capacity due to peripheral congestion, which can improve container properties, and also can allow the bulk incorporation of bioactive molecules of higher molecular weight and of different chemistry. Simultaneously, these nanocarriers can maintain the high internalization efficiency with improved biological properties, when compared to conventional dendrimers. ${ }^{[23]}$

Following on this initial report, the aim of this work consisted in determining the possible application of the CMCht/PAMAM dendrimer nanoparticles for CNS-related applications. Our future objective will be to use them as intracellular cell targeted systems for the delivery of relevant drugs trough local injection on SCI sites. For this initial study we investigated: (i) the effects CMCht/PAMAM dendrimer nanoparticles in neuronal and glial cell viability and (ii) the internalization of the CMCht/PAMAM dendrimer nanoparticles by CNS-derived cells. Results revealed that the CMCht/PAMAM dendrimer nanoparticles were not deleterious to both hippocampal neurons and cortical glial cells. Furthermore, it was also possible to observe that neurons, astrocytes, oligodendrocytes and microglial cells were able to internalize the CMCht/PAMAM dendrimer nanoparticles. The latter was different according to the cell population observed, but overall started after $1 \mathrm{~h}$ of incubation reaching a peak, that ranged between 80 and $100 \%$, after 48 h of exposure. Moreover, it was also observed, namely for oligodendrocytes and neurons, that the periodical addition of CMCht/PAMAM dendrimer nanoparticles was required to maintain the percentage of positive cells for internalization of CMCht/PAMAM dendrimer nanoparticles for periods of up to $7 \mathrm{~d}$ in culture.

\section{Experimental Part}

\section{CMCht/PAMAM Nanoparticle Synthesis}

The synthesis of the CMCht/PAMAM dendrimer nanoparticles was carried out as described in ref. ${ }^{\left[{ }^{30]}\right.}$ Firstly, CMCht was synthesized starting from chitosan of medium molecular weight (Aldrich, USA). Then, CMCht/PAMAM dendrimer nanoparticles were prepared in a step-by-step method, as follows: (i) increasing the generation of the poly(amidoamine) carboxylic-terminated dendrimers of generation 1.5 (PAMAM-CT, G1.5), (ii) obtaining a PAMAM methyl ester terminated dendrimer, (iii) reaction of PAMAM with carboxymethylchitosan possessing a degree of deacetylation of $80 \%$ and degree of substitution of $47 \%$. In brief, PAMAM-CT (G1.5) was dissolved in ultra-pure water and 1-ethyl-(3-dimethylaminopropyl) carbodiimide hydrochloride (EDC, Fluka, Slovakia) was then added to the solution at a molar ratio sufficient to modify the carboxylate residue of the dendrimers. The $\mathrm{pH}$ was maintained at 6.5, under agitation and after $30 \mathrm{~min}$, ethylenediamine (EDA, Sigma, Germany) was added to the solution at a molar ratio equal to that of EDC. After purification by dialysis, the PAMAM methyl ester dendrimer was obtained by adding an appropriate volume of methyl methacrylate (Fluka, Germany) to the PAMAM amine- 
terminated compound. Then, the CMCht/PAMAM dendrimer nanoparticles with carboxylic-terminated groups were produced by mixing CMCht powders $(100 \mathrm{mg}$ ) and the PAMAM methyl ester dendrimer $(50 \mathrm{mg}$ ) in a water/methanol solution for $72 \mathrm{~h}$ (reductive $\mathrm{N}$-alkylation). After this period, the CMCht/PAMAM dendrimer nanoparticles were precipitated by adding an appropriate volume of a saturated sodium carbonate, $\mathrm{Na}_{2} \mathrm{CO}_{3}$ (Aldrich, Germany) solution and acetone (Pronalab, Portugal). Precipitates were collected by filtration, dispersed in ultra-pure water for extensive dialysis and freeze-dried (Telstar-Cryodos -80 , Spain) at $-80{ }^{\circ} \mathrm{C}$.

In order to trace down the CMCht/PAMAM dendrimer nanoparticles within the intracellular compartment they were also labeled with fluorescein isothiocyanate (FITC) (Sigma, Germany). For this purpose conjugates of CMCht/PAMAM-FITC were prepared by covalently bonding the amine group of carboxymethylchitosan and the isothiocyanate group of FITC (thiourea bond). Briefly, a CMCht/PAMAM dendrimer nanoparticle solution was prepared by dissolving the nanoparticles in a carbonate/bicarbonate coupled buffer of $\mathrm{pH}=9.2$ at a final concentration of $10 \mathrm{mg} \cdot \mathrm{mL}^{-1}$. Then, $50 \mu \mathrm{L}$ of FITC/dimethyl sulfoxide (DMSO) solution was added per $1 \mathrm{~mL}$ of CMCht/PAMAM dendrimer nanoparticle buffered solution, and the mixture was kept under agitation for the period of $8 \mathrm{~h}$ at $4{ }^{\circ} \mathrm{C}$ (protected from light). Finally, the FITC-labeled CMCht/PAMAM dendrimer nanoparticle solution was dialyzed in order to remove the unbound FITC, freezed at $-80^{\circ} \mathrm{C}$ and freeze-dried. The labeling efficiency was investigated by UV-Vis spectrophotometry (NanoDrop ND-1000; NanoDrop Technologies, USA) by reading the absorbance at 280 and $495 \mathrm{~nm}$, and the determination of FITC:CMCht/PAMAM dendrimer nanoparticles molar ratio calculated using a standard curve.

\section{Physical Characterization of CMCht/PAMAM Dendrimer Nanoparticles}

The morphology of the nanoparticles was investigated using atomic force microscopy (AFM). The freeze-dried CMCht/PAMAM dendrimer nanoparticles were dispersed in ultrapure water to obtain a solution with final concentration of $1 \mathrm{mg} \cdot \mathrm{mL}^{-1}$, and then $20 \mu \mathrm{L}$ was placed over a $9.9 \mathrm{~mm}$ mica disc (Agar Scientific, England) and blown dried with nitrogen gas for subsequent characterization. The samples were analyzed using the Tapping Mode with a MultiMode AFM connected to a NanoScope III controller, both from Veeco, USA, with non-contact silicon nanoprobes (ca $300 \mathrm{kHz}$ ) from Nanosensors, Switzerland. All images were plane-fitted using the third degree-flatten procedure included in the NanoScope software version 4.43r8. The morphometric analysis was performed using the same software. In addition, the morphology of the particles was analyzed under a transmission electron microscope (TEM, Philips CM-12, FEI Company, The Netherlands, equipped with a MEGA VIEW-II DOCU camera and Image Software Analyzer SIS NT DOCU). For the purpose of TEM studies, a solution of $1 \mathrm{mg} \cdot \mathrm{mL}^{-1} \mathrm{CMCht} / \mathrm{PAMAM}$ dendrimer nanoparticles solution was prepared using ultrapure water and then stained with $2 \%$ phosphotungstic acid. The stained nanoparticles solution was transferred to copper grids for further observation.

\section{In vitro Studies}

\section{Primary Cultures of Hippocampal Neurons}

Hippocampal neuron cultures were prepared from P4 Wistar rats. ${ }^{[31]}$ Upon dissection, hippocampi tissue samples were submitted to a $10 \mathrm{~min}$ ' trypsin-based enzymatic digestion $(0.05 \%$ Trypsin - Gibco; $3 \mathrm{mg} \cdot \mathrm{mL}^{-1}$ bovine serum albumin, Sigma; 0.0025\% DNase - Sigma, in Earle's Balanced Salt Solution (EBSS), Gibco) followed by mechanical dissociation and centrifugation. Isolated cells were then plated on poly(D-lysine)-coated coverslips at a density of $4 \times 10^{4}$ cells $\cdot \mathrm{cm}^{-2}$. Cultures were maintained $\left(37^{\circ} \mathrm{C}\right.$, $5 \% \mathrm{CO}_{2} / 95 \%$ air, $90 \%$ relative humidity) in Neurobasal A medium/ B27 supplemented with $10^{-3} \mathrm{M}$ Glutamax I, $10 \mathrm{ng} \cdot \mathrm{mL}^{-1}$ basic fibroblast growth factor (b-FGF) and $0.1 \mathrm{mg} \cdot \mathrm{mL}^{-1}$ kanamycin (all from Gibco, USA) for 7 days. From day 7 onwards two sets of experiments were performed, as follows.

In order to determine the internalization time-periods of FITClabeled CMCht/PAMAM dendrimer nanoparticles by hippocampal neurons, cultures were incubated with $200 \mu \mathrm{g} \cdot \mathrm{mL}^{-1}$ of nanoparticles for periods of $1,2,6,12,15,18,24$ and $48 \mathrm{~h}$ ( $n=3$ /time-point). After these time-points cells were immunostained for neuronal markers and observed under a fluorescence microscope. The criteria established for positive cells for nanoparticles internalization was the observation of FITC-labeled CMCht/PAMAM dendrimer nanoparticles within the intracellular compartment.

A second set of experiments was also conducted with the aim of assessing if the percentage of positive cells for FITC-labeled CMCht/ PAMAM internalization throughout time was dependent, or not, on the maintenance of the nanoparticles concentration in the culture medium. For this purpose cultures were incubated for periods up to 7 days in which nanoparticles were supplemented to the culture medium every $48 \mathrm{~h}$ in one group $(n=3)$, while in the other nanoparticles were only added on the first day of the experiment $(n=3)$. The same criterion as above was used to determine positive cells for FITC-labeled CMCht/PAMAM dendrimer nanoparticle internalization. For the identification of positive cells, cultures were immunostained for neuronal markers and observed under a fluorescence microscope.

\section{Primary Cultures of Cortical Glial Cells}

Cortical glial cells were isolated from P4 newborns (Wistar Rats) as previously described in ref. ${ }^{[32]}$ Upon dissection, cortices were submitted to an enzymatic cocktail $\left(30 \mathrm{mg} \cdot \mathrm{mL}^{-1}\right.$ DNase, $0.25 \%$ Trypsin, both from Sigma) in $\mathrm{Ca}^{2+} / \mathrm{Mg}^{2+}$-free Hanks' Balanced Salt Solution (HBSS) (Gibco) followed by mechanical dissociation. Glial cells were then obtained by centrifuging the resulting cell suspension at $800 \mathrm{rpm}$ for $2 \mathrm{~min}$, and plated out on coverslips previously coated with poly(D-lysine) at a density of $4 \times 10^{4}$ cells $\cdot \mathrm{cm}^{-2}$. Glial cells were then maintained for 1 week in Dulbecco's modified eagle medium (Sigma) supplemented with $1 \%$ antibiotics/antimycotics solution (Sigma, USA) and 10\% FBS. At this time point similar experiments to those performed with hippocampal cultures were carried out $(n=3)$. In order to determine if the astrocyte and oligodendrocyte cells proliferation was affecting the percentage of positive cells internalizing the nanoparticles, after $7 \mathrm{~d}$ of incubation with or without renovation, cultures were treated at day 7 with 5 -fluoro-2'- 
deoxiuridina (FDU, Sigma), a known inhibitor of glial cell proliferation.

\section{Cell Viability Assessment}

For both neurons and glial cells, cell viability was assessed by the MTS [3-(4,5-dimethylthiazol-2-yl)-5-(3-carboxymethoxyphenyl)2(4-sulfophenyl)-2H-tetrazolium] test (Promega, USA) after $7 \mathrm{~d}$ of incubation (with or without renovation) with FITC-labeled CMCht/ PAMAM dendrimer nanoparticles. The test is an assay in which the substrate MTS is bioreduced into a brown formazan product by NADPH or NADP produced by mitochondrial enzymes, which are active in living cells. ${ }^{[3,34]}$ This assay, or others similar to it (MTT, WST-1), have been widely used to measure cellular viability and proliferation. Hippocampal neurons and glial cells cultures incubated under the conditions previously described $(n=3)$ were placed in culture medium containing MTS in a 5:1 ratio and incubated in a humidified atmosphere at $37{ }^{\circ} \mathrm{C}$ and $5 \% \mathrm{CO}_{2}$. After $3 \mathrm{~h}$ of incubation, $100 \mu \mathrm{L}$ of solution from each well were transferred to 96-well plates and the optical density was determined at $490 \mathrm{~nm}$. Cultures that were not exposed to the FITC-labeled CMCht/PAMAM dendrimer nanoparticles were used as controls.

\section{Immunocytochemistry}

Following each culture period, the cells were fixed in $4 \%$ paraformaldehyde for $30 \mathrm{~min}$, permeabilized by incubation with $100 \%$ methanol for $2 \mathrm{~min}$ at room temperature, and washed three times in PBS. Cells were blocked with $10 \%$ fetal bovine serum/ phosphate-buffered saline $(60 \mathrm{~min})$, followed by a $60 \mathrm{~min}$ incubation with primary antibodies: mouse anti-rat microtubule associated protein-2 (MAP-2) (Sigma, USA, 1:500) to detect mature hippocampal neurons, rabbit anti-rat glial fibrillary acid protein (GFAP) (Dako, Denmark, 1:500) for astrocytes, mouse anti-CD11b (BD Biosciences Pharmigen, USA, 1:100) for microglial cells and mouse anti-O4 (R\&D systems, USA, 1:50) for oligodendrocytes. Finally, cells were washed in PBS and incubated with the secondary antibody: Alexa Fluor 594 goat anti-mouse immunoglobulin G (IgG) and Alexa Fluor goat anti-rabbit 488 (Molecular Probes, USA) for $60 \mathrm{~min}$. The primary antibody was omitted to prepare the negative controls. Samples were further observed under an Olympus BX-61 Fluorescence Microscope (Olympus, Germany). The percentage of FITC-labeled CMCht/PAMAM dendrimer nanoparticles internalization by the different cell populations was achieved by the following ( $n=3,5$ random fields/coverslip): \% of positive cells for internalization $=$ number of cells positive for FITClabeled CMCht/PAMAM dendrimer nanoparticles internalization/ total number of cells.

\section{Statistical Analysis}

Statistical evaluation was performed using one way ANOVA (internalization time periods experiments) and two-tailed paired $t$ student test (MTS, comparison between groups in which CMCht/ PAMAM dendrimer nanoparticles were added every $48 \mathrm{~h}$ or just added on the first day of the experiment), to assess the statistical differences between different groups. Statistical significance was defined as $p<0.05$ for a $95 \%$ confidence interval.

\section{Results and Discussion}

In the present study, we aimed at determining the interactions between a new class of nanoparticle based systems, known as CMCht/PAMAM dendrimer nanoparticles, with hippocampal neurons and cortical glial cells in order to develop future strategies for the delivery of therapeutic drug in CNS-regenerative medicine. For this purpose, CMCht/PAMAM dendrimer nanoparticles were firstly characterized under AFM and TEM, after which hippocampal neurons and cortical glial cells were exposed to the above-referred nanoparticles for different culture periods.

In order to further characterize the CMCht/PAMAM dendrimer nanoparticles an AFM study was performed to both evaluate their size and morphology (Figure 1). From Figure 1A, it is possible to observe the 3-D AFM image of the PAMAM-CT (G1.5) dendrimers. This data reveal the monodispersive character of these macromolecules. On the other hand, Figure $1 \mathrm{~B}$ shows the 3-D image of the CMCht/PAMAM dendrimer nanoparticles, which clearly shows the consistency of these molecules, i.e., nanosphere-like shape. By performing a threshold technique it became possible to perform their morphometric analysis. This study revealed that the CMCht/PAMAM dendrimer nanoparticles have a mean size ranging from 22.0 to $30.7 \mathrm{~nm}$ for a confidence level of $95 \%$, and a confidence level of \pm 4.4 (Figure 1D). Dynamic light scattering studies revealed that CMCht/ PAMAM dendrimer nanoparticles present a size of approximately $45 \pm 15$ and $250 \pm 100 \mathrm{~nm}$, in aqueous solution. As previously reported $^{[30]}$ the peak at $\approx 45 \mathrm{~nm}(36.2 \%)$ corresponds to the isolated CMCht/PAMAM dendrimer nanoparticles, and the peak at $\approx 250 \mathrm{~nm}$ is attributed to the aggregated nanoparticles. This data were further confirmed by TEM analysis (Figure 1E) that revealed the nanospherelike morphology of the nanoparticles. CMCht/PAMAM dendrimer nanoparticles were then labeled with FITC to allow qualitative and quantitative evaluation of internalization. The labeling efficiency and stability were investigated by UV-Vis spectrophotometry. Figure $1 \mathrm{~F}$ shows the UV-Vis spectrum of the FITC-labeled CMCht/ PAMAM dendrimer nanoparticles. The FITC:CMCht/ PAMAM dendrimer nanoparticles ratio was 1:1.1.

Regarding the biological assays, the effects of the CMCht/ PAMAM dendrimer nanoparticles on neurons and glial cells viability were the first to be assessed. For this purpose both cell cultures systems were incubated for 1 week with the nanoparticle based system under study. In one of the groups CMCht/PAMAM dendrimer nanoparticles concentration was renewed every $48 \mathrm{~h}$, while in the other, nanoparticles 
A

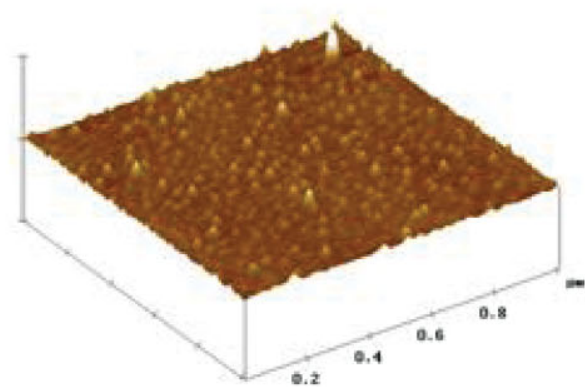

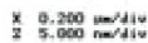

B

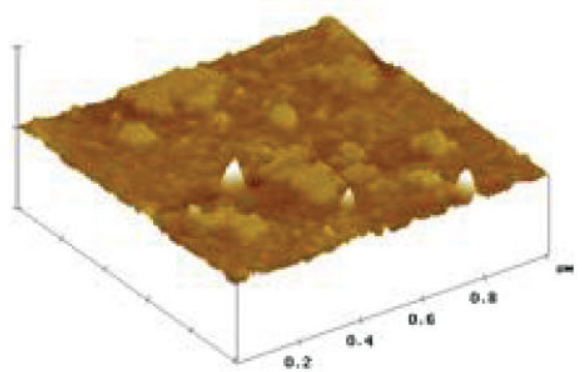

$\frac{x}{2} \frac{0,200}{5,000}=0$
C

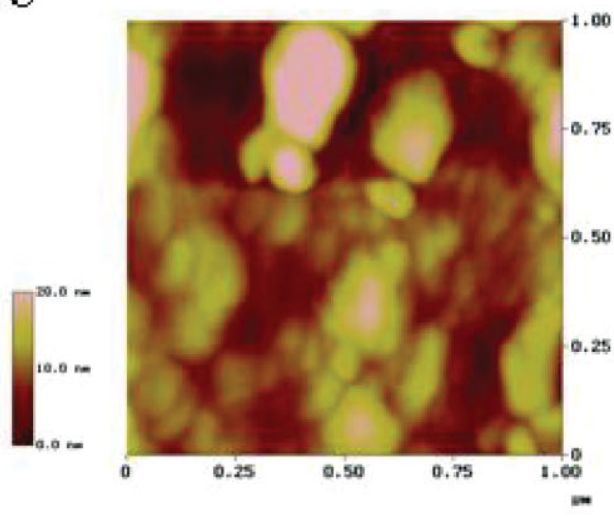

D

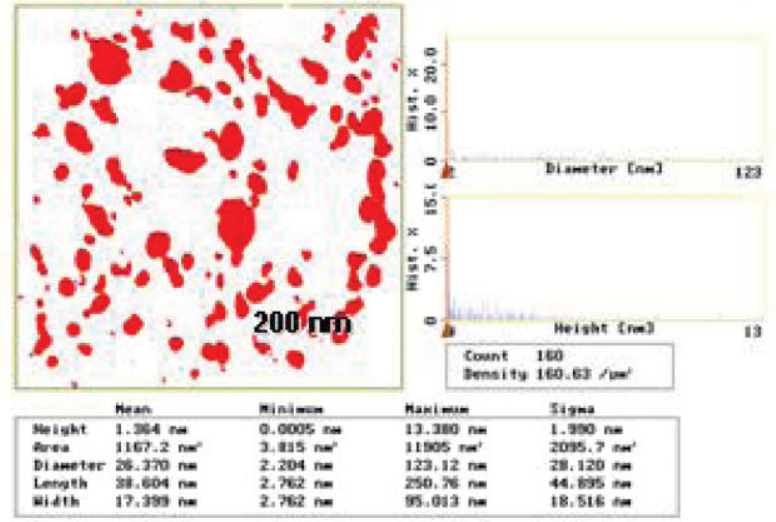

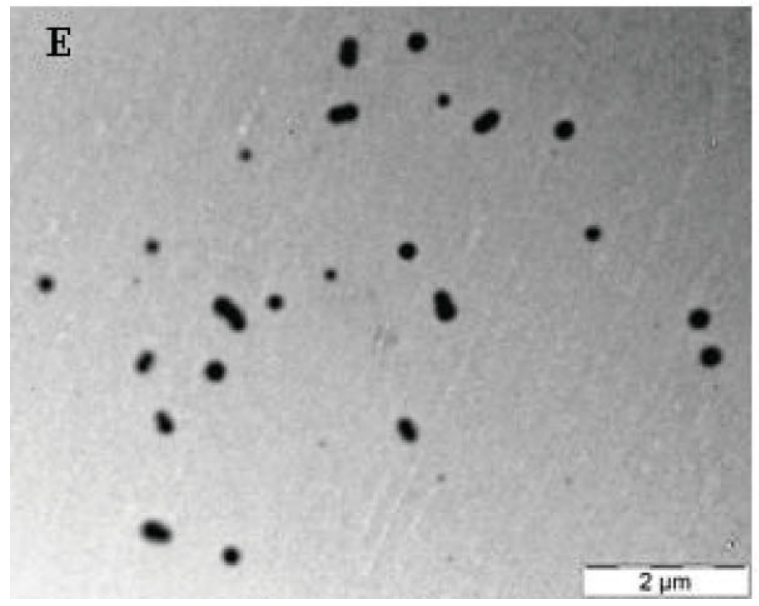

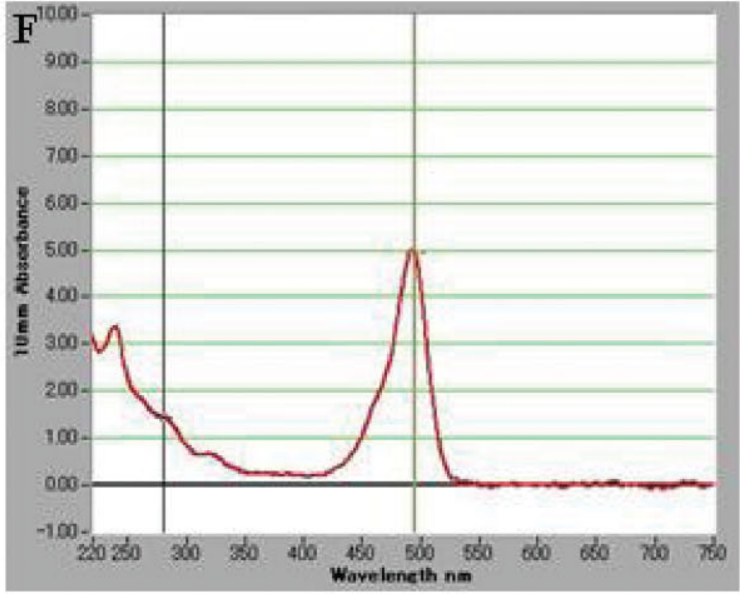

Figure 1. FITC-labeled CMCht/PAMAM dendrimer nanoparticles. (A) AFM 3-D images of the PAMAM-CT, G1.5; (B) CMCht/PAMAM dendrimer nanoparticles spread on a mica surface after the solute (water) being wick off with filter paper; (C) AFM 2-D image of the CMCht/PAMAM dendrimer nanoparticles and (D) respective morphometric analysis; (E) Transmission electron microscope micrograph of the CMCht/ PAMAM dendrimer nanoparticles; (F) UV-Vis spectrum of FITC-labeled CMCht/PAMAM dendrimer nanoparticles.

were only added to the culture medium at day 0 of the experiment with no further addition. Results for the MTS test revealed that both cell populations were producing large amounts of a brown formazan product which is an indicator of normal cell viability and metabolism, as cells were able to incorporate and metabolize MTS in similar values when compared to those obtained for the controls $(\approx 100 \%$ for the values obtained for positive control for cell viability). Additionally, no significant differences were found between the two different groups where CMCht/ PAMAM dendrimer nanoparticles were added $(p>0.05)$. Therefore it was found that CMCht/PAMAM dendrimer nanoparticles were non-cytotoxic for both post-natal hippocampal neurons and glial cells.

After screening the possible cytotoxicity of CMCht/PAMAM dendrimer nanoparticles, the objectives of the present report 
were focused on the determination of the internalization time-periods and percentage of cells internalizing the nanoparticles in two different sets of experiments.

Figure 2 shows the results obtained in primary cultures of post-natal hippocampal neurons. As it can be observed hippocampal neurons were able to internalize the FITClabeled CMCht/PAMAM dendrimer nanoparticles (Figure 2A, after $24 \mathrm{~h}$ of exposure to a concentration of $200 \mu \mathrm{g} \cdot \mathrm{mL}^{-1}$ ). Furthermore, this study also revealed that the percentage of positive cells internalizing the FITC-labeled CMCht/PAMAM dendrimer nanoparticles tended to increase with culturing time, presenting two different periods (Figure 2B): (i) between 1 and $3 \mathrm{~h}$ (around 5-10\%); (ii) between 6 and $48 \mathrm{~h}$, with increasing values that were around $70 \%$ after $48 \mathrm{~h}$. Groups $1-3 \mathrm{~h}$ presented statistically significant differences $(p<0.05)$ in respect to internalization when compared to groups 15 to $48 \mathrm{~h}$. Another feature noticed with these experiments was the high variability (high standard
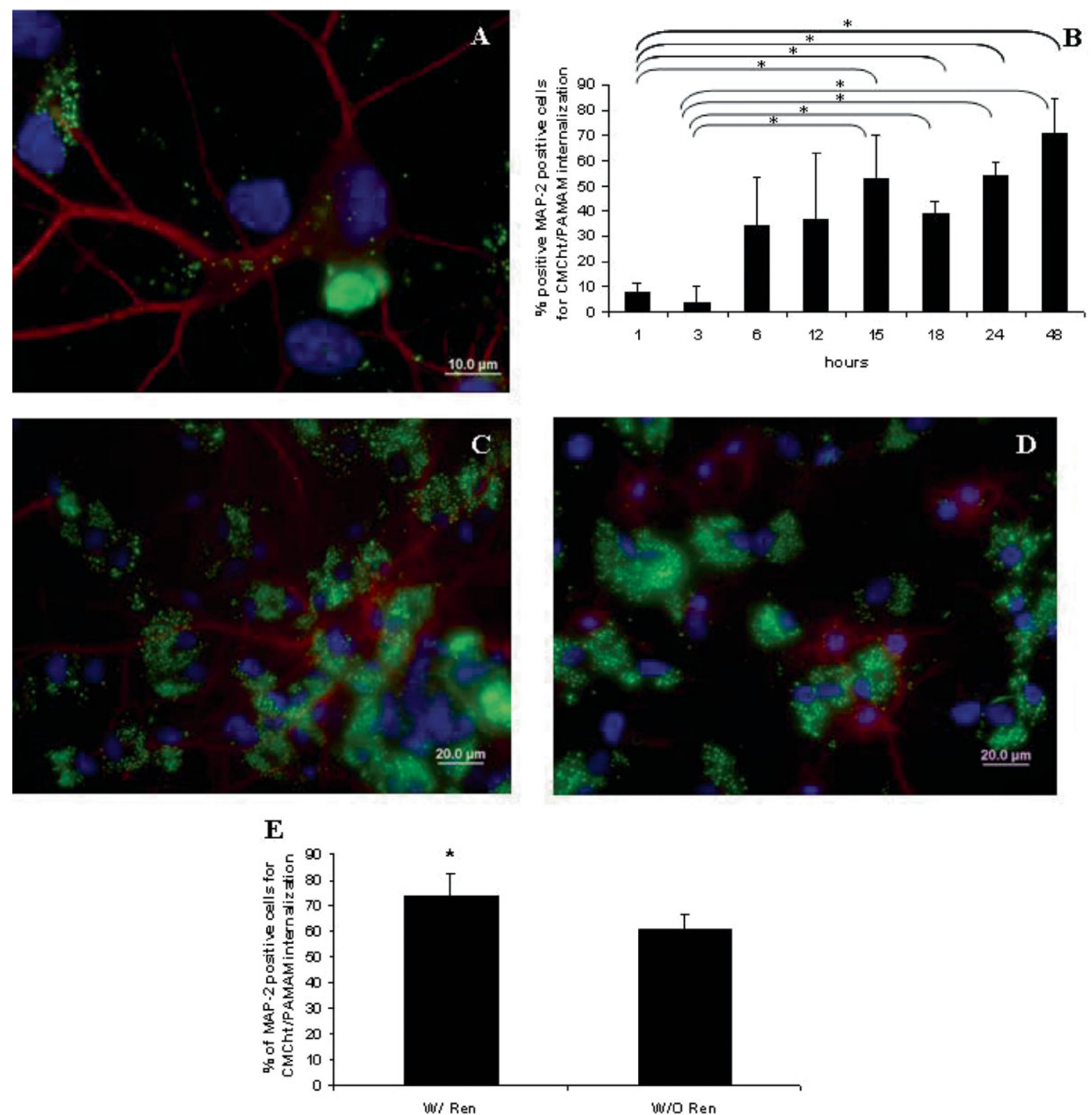

Figure 2. Postnatal hippocampal neurons were able to internalize the FITC-labeled CMCht/PAMAM dendrimer nanoparticles (A). Initially neuronal cells disclosed a high variability on the capacity of internalizing the nanoparticles. However results tended to be more homogeneous after $24 \mathrm{~h}$ of incubation, reaching a maximum of internalization (around $80-90 \%$ ) after $48 \mathrm{~h}$ (B). Further experiments (C-E; C-with renovation and D-without renovation) also showed that the maintenance of the CMCht/PAMAM dendrimer nanoparticles concentration in the culture medium was essential to maintain the percentage of positive cells internalizing the nanoparticles. The differences observed in C-E were attributed to the proliferation of other cell populations within the hippocampal neuron cultures, namely neuroprogenitor cells ( $n=3 ; 5$ fields/coverslip; mean \pm SD; $p<0.05$ ). 
deviation) obtained up to the $12 \mathrm{~h}$ time point (Figure 2B). This fact is an indication that not all hippocampal neurons disclosed the same response at earlier incubation time points. However, as it can be observed in Figure 2B, this behavior fades way with time. After 24 and $48 \mathrm{~h}$ the obtained results were much more homogeneous, as it can be seen by the indicative values of the standard deviation bars. Finally, it was also observed that the periodical addition of the CMCht/PAMAM dendrimer nanoparticles in the culture medium is essential for the maintenance of the internaliza- tion percentage by hippocampal neuronal populations (Figure 2C-E, $p<0.05$ ). It is believed that this fact is related with the proliferation of other cell populations present in this culture system, namely neuroprogenitor cells.

Similar to what it can be observed for hippocampal neurons, all three glial cell types were able to internalize the CMCht/PAMAM dendrimer nanoparticles (Figure 3-5). This observation is noteworthy to mention, as in the culture system used in the present work all glial cell types were present, and therefore it is an indication that the
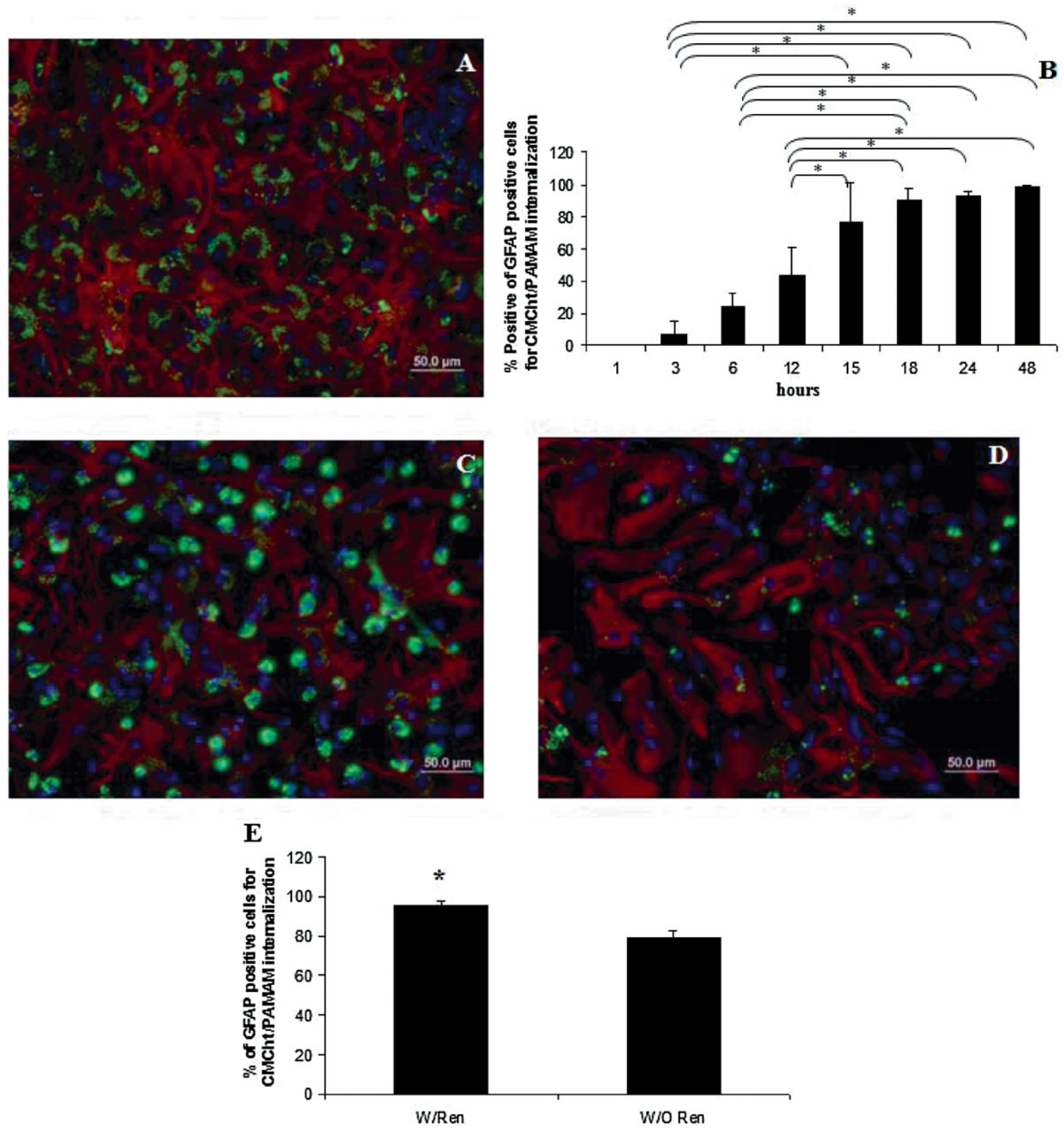

Figure 3. Internalization experiments within cortical glial cell cultures showed that astrocytes were also able to internalize the FITC-labeled CMCht/PAMAM dendrimer nanoparticles (A- after $48 \mathrm{~h}$ of incubation). As it can be observed in (B) the internalization occurred in a gradual manner reaching a peak (around $100 \%$ of internalization) after $48 \mathrm{~h}$ of incubation. When exposed for longer periods of time it was observed that the periodical addition of nanoparticles to the culture medium was needed to stabilize the percentage of positive cells internalizing the nanoparticles (C-E; C with and D without renovation). ( $n=3 ; 5$ fields/coverslip; mean $\pm \mathrm{SD} ; p<0.05$ ). 

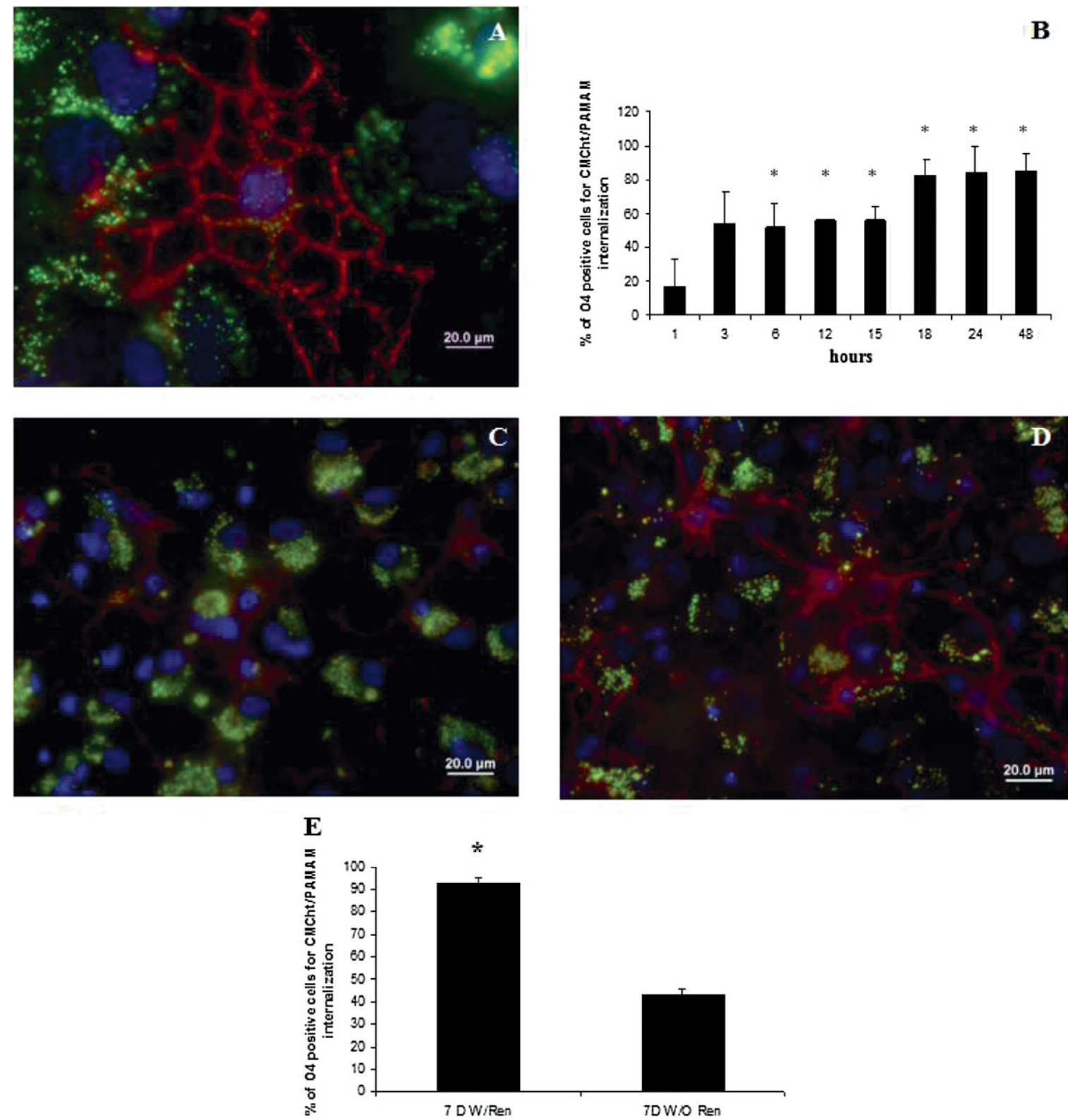

Figure 4. Oligodendrocytes also reveal to be capable of internalizing the FITC-labeled CMCht/PAMAM dendrimer nanoparticles. (A) Representative image of the nanoparticles distributed along the intracellular compartment. As it can be observed they were scattered around the nucleus, as well as distributed along the cytoplasmatic processes of the cells. Within the oligodendrocytes population it was possible to observe that the highest value of internalization was obtained after $48 \mathrm{~h}$ of incubation (B-around $80-90 \%$ ). Similarly to what happened with neurons and astrocytes, the maintenance of the percentage of positive cells internalizing the nanoparticles within the oligodendrocyte cell population was dependent on the maintenance of the concentration of the FITC-labeled CMCht/PAMAM dendrimer nanoparticles in the culture medium (C-E; C with and D without renovation). ( $n=3 ; 5$ fields/coverslip; mean $\pm \mathrm{SD} ; p<0.05)$.

nanoparticles under study are not preferentially up taken and consequently phagocytized by microglial cells (Figure 3A, 4A, 5A). Nevertheless, all glial cell populations disclosed different internalization patterns. For instance, astrocytes (Figure 3) revealed a steady increase on the number of positive cells for CMCht/PAMAM dendrimer nanoparticles internalization reaching the highest value after $48 \mathrm{~h}$ of incubation (around 100\%, Figure 3B). On the other hand oligodendrocytes (Figure 4) disclosed three different levels of internalization, with the highest value being around 80-90\% (Figure 4B). Finally, microglia cells (Figure 5) had the fastest and highest rates of internalization, showing levels of around $100 \%$ after $12 \mathrm{~h}$ of incubation (Figure 5B). This observation can be explained due to microglia cells phagocytotic nature. The differences obtained between astrocytes and oligodendrocytes to the 

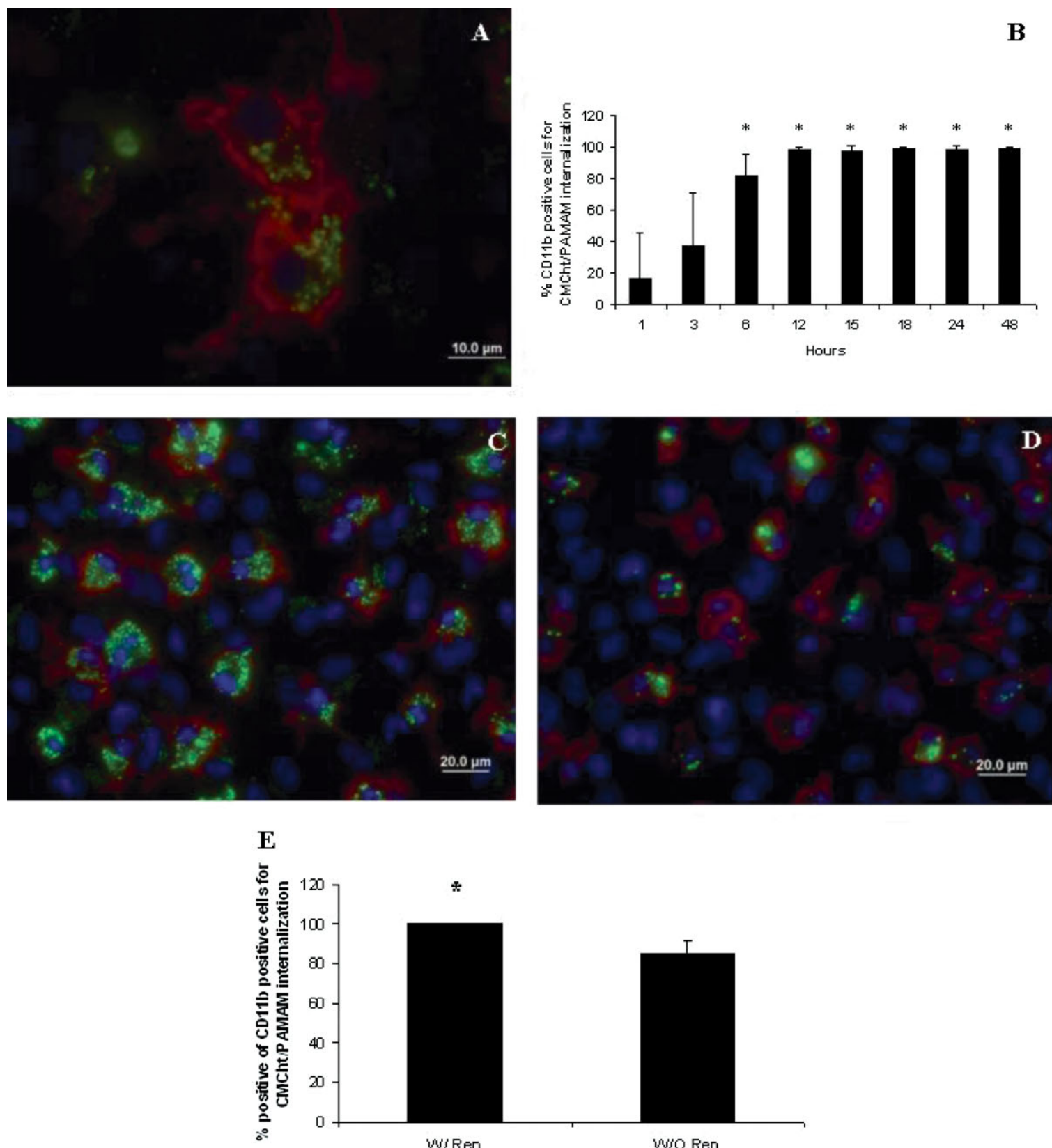

E
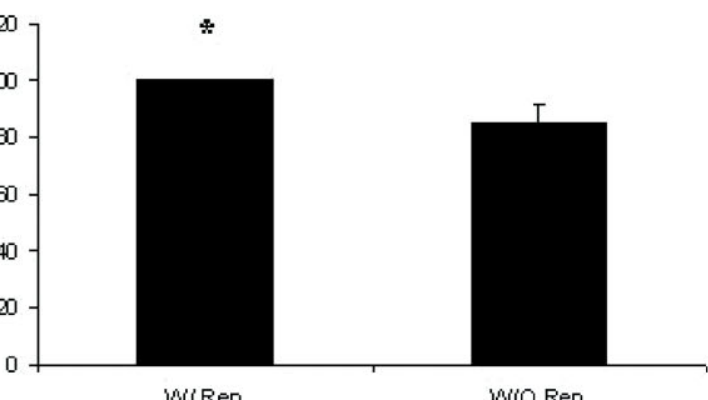

Figure 5. Microglial cells were able to internalize FITC-labeled CMCht/PAMAM dendrimer nanoparticles at a faster rate (A, B) as compared to other neuronal and glial cell populations. This fact cannot be dissociated from the phagocytotic capability of these cells. Similar effects to those previously observed with other cell populations regarding the influence of the periodical addition of nanoparticles on the percentage of positive cells internalizing the nanoparticles (C-E; C with and D without renovation). This result is probably related with the macrophagelike behavior of these cells.

same concentration of FITC-labeled CMCht/PAMAM dendrimer nanoparticles are probably related with their different endocytotic capability, as a consequence of the different functions that they have in the CNS.

Similarly to what was observed in hippocampal neuronal cultures, the percentage of positive cells for FITC-labeled CMCht/PAMAM dendrimer nanoparticles internalization was also reduced for astrocytes $(p<0.05)$, microglial cells $(p<0.05)$ and oligodendrocytes $(p<0.05)$, whenever the nanoparticles were not added every $48 \mathrm{~h}$ to the culture media. As microglial cells do not proliferate in this culture system $^{[32]}$ the observed results might be attributed to their phagocytic nature and high content of degrading enzymes, which might be leading to the degradation of the nanoparticle based systems. However this needs to be further investigated in forthcoming studies. However, for astrocytes and oligodendrocytes the observed phenomenon can be related to cell proliferation, as these cells do proliferate 

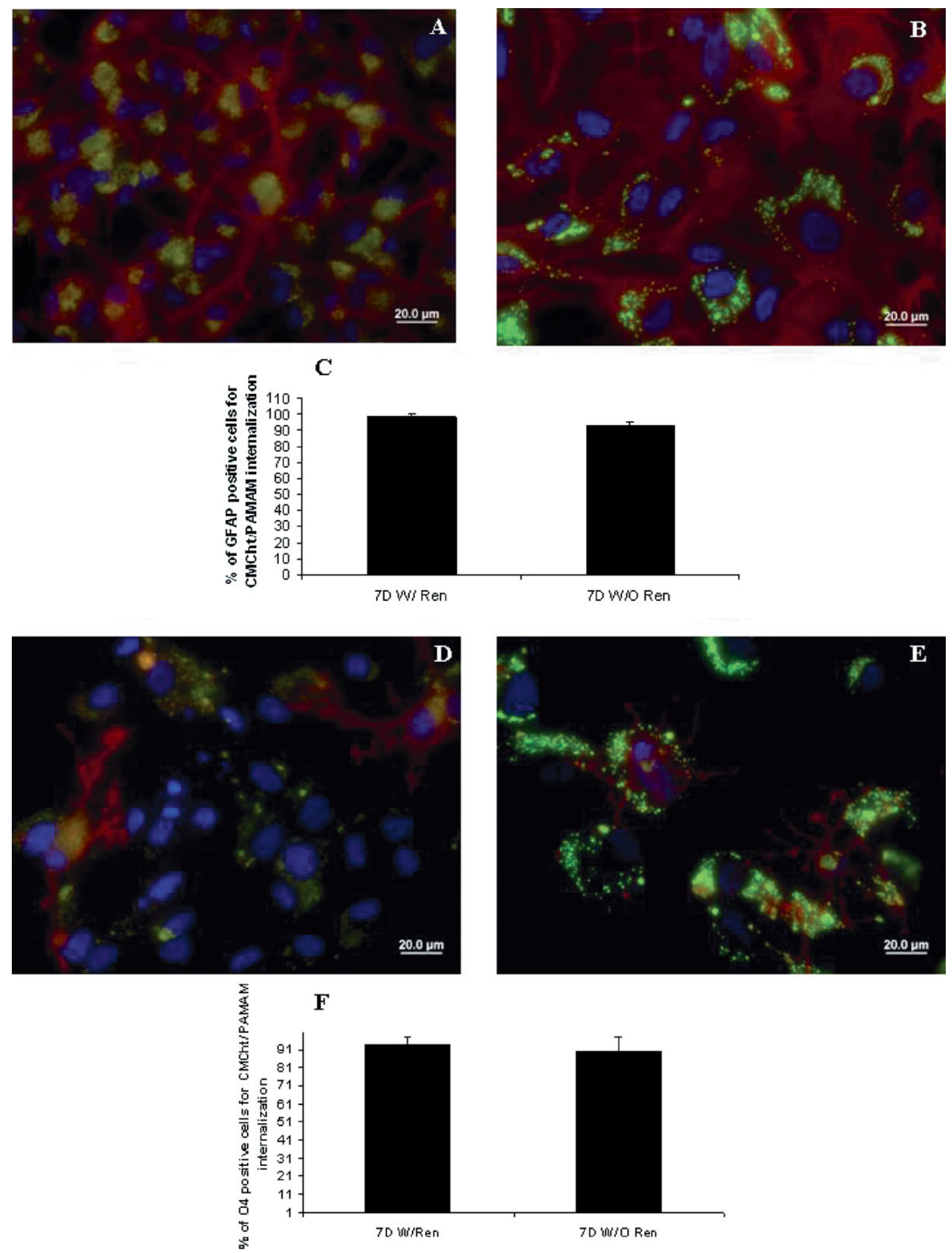

Figure 6. Cortical glial cells cultures were treated with FDU in order to determine if the decrease on the rate of FITC-labeled CMCht/PAMAM dendrimer nanoparticles internalization by astrocytes $(A-C)$ and oligodendrocytes $(D-F)$ was related to their cell proliferation. As it can be observed, whenever astrocyte and oligodendrocyte cells proliferation is impaired, the levels of internalization are not dependent on the periodical addition of FITC-labeled CMCht/PAMAM dendrimer nanoparticles to the culture medium. This fact is an indication that the results observed in Figure 4 and 5 are related with the cell proliferation of the above referred cell populations. 
in this culture system. This hypothesis was further assessed by stopping cell proliferation within the cortical glial cell primary cultures (Figure 6). In these culturing conditions, and as it can be observed in Figure 6, no differences were found between the two tested groups $(p>0.05)$. In this sense one might consider that, for now, these nanoparticles may be considered for short term applications, so cell proliferation does not play a major role on their possible therapeutic capability.

\section{Conclusion}

With the present work it was possible to demonstrate that carboxymethylchitosan/poly(amidoamine) dendrimer nanoparticles disclosed a nanosphere-like shape. Furthermore it was also revealed that the binding of these nanoparticles to fluorescent probes (e.g., FITC) for tracing purposes was also possible. At the biological level, we were able to determine that the cell viability of post-natal neurons and glial cells was not affected upon exposure to these nanoparticles. Moreover, it was possible to observe that neurons, astrocytes, oligodendrocytes and microglial cells were able to internalize, at different rates, the CMCht/PAMAM dendrimer nanoparticles. Finally, it was also observed that cell proliferation of astrocytes and oligodendrocytes lead to a decrease on the levels of internalization within their total cell populations. Further studies will be focused on loading relevant drugs for CNS-related applications (e.g., SCI) into the CMCht/PAMAM dendrimer nanoparticles.

Acknowledgements: Financial support from the Portuguese Foundation for Science and Technology through funds from POCTI and/or FEDER programs (Funding to ICVS, 3B's Research Group, post-doctoral and pre-doctoral fellowships to A. J. Salgado and J. M. Oliveira - SFRH/BPD/17595/2004; SFRH/BD/21786/2005) is gratefully acknowledged. This work was also carried out under the scope of the European NoE EXPERTISSUES (NMP3-CT-2004-500283) and HIPPOCRATES STREP (NMP3-CT-2003-505758) projects.

Received: January 5, 2010; Revised: March 17, 2010; Published online: July 2, 2010; DOI: 10.1002/mabi.201000005

Keywords: cell viability; dendrimers; glial cells; internalization; intracellular drug delivery systems

[1] B. K. Kwon, W. Tetzlaff, J. N. Grauer, J. Beiner, A. R. Vaccaroi, Spine J. 2004, 4, 451.

[2] W. L. Liu, Y. H. Lee, S. Y. Tsai, C. Y. Hsu, Y. Y. Sun, L. Y. Yang, S. H. Tsai, W. C. Yang, Glia 2008, 56, 1390.

[3] F. Duksal, I. Kilic, A. C. Tufan, I. Akdogan, Brain Res. 2009, 1250,75 .
[4] J. R. Kanwar, G. Mahidara, R. K. Kanwar, Curr. Nanosci. 2009, $5,441$.

[5] G. Orive, E. Anitua, J. L. Pedraz, D. F. Emerich, Nat. Rev. Neurosci. 2009, 10, 682

[6] G. Modi, V. pillay, Y. E. Choonara, Y. M. K. Ndesendo, L. C. du Toit, D. Naidoo, Prog. Neurobiol. 2009, 88, 272.

[7] E. Barbu, E. Molnar, J. Tsibouklis, D. C. Gorecki, Exp. Opin. Drug Delivery 2009, 6, 553.

[8] S. Shinoda, M. Ohashi, H. Tsukube, Chem. Eur. J. 2007, 13, 81.

[9] M. Hussain, M. S. Shchepinov, M. Sohail, I. F. Benter, A. J. Hollins, E. M. Southern, S. Akhtar, J. Contam. Relat. 2004, 99, 139.

[10] C. Z. Chen, S. L. Cooper, Adv. Mater. 2000, 12, 843.

[11] N. Sousa, J. J. Cerqueira, O. F. Almeida, Brain Res. Rev. 2008, 57, 561.

[12] J. Kreuter, R. N. Alyautdin, D. A. Kharkevich, A. A. Ivanov, Brain Res. 1995, 674, 171.

[13] R. N. Alyautdin, V. Petrov, K. Langer, A. Berthold, D. A. Kharkevich, J. Kreuter, Pharm. Res. 1997, 14, 325.

[14] A. E. Gulyaev, S. E. Gelperina, I. N. Skidan, A. S. Antropov, G. Y. Kivman, J. Kreuter, Pharm. Res. 1999, 16, 1564.

[15] J. Kreuter, P. Range, V. Petrov, S. E. Hamm Gelperina, B. Engelhardt, R. Alyautdin, H. von Briesen, D. J. Begley, Pharm. Res. 2003, 20, 409.

[16] N. Shi, Y. Zhang, C. Zhu, R. J. Boado, W. M. Partdrige, PNAS. 2001, 98, 12754

[17] C. Jiang, N. Koyabu, Y. Yonemitsu, T. Shimazoe, S. Watanabe, M. Naito, T. Tsuruo, H. Ohtani, Y. Sawada, Hum. Gene Ther. 2003, 14, 1181.

[18] M. T. da Cruz, S. Simões, M. C. de Lima, Exp. Neurol. 2004, 187, 65.

[19] M. T. da Cruz, A. L. Cardoso, L. P. de Almeida, S. Simões, M. C. de Lima, Gene Ther. 2005, 12, 1242.

[20] G. Tosi, L. Constantinp, F. Rivasi, B. Ruozi, E. Leo, A. V. Vergoni, R. Tacchi, A. Bertolini, M. A. Vandelli, F. Forni, J. Controlled Release 2007, 122, 1.

[21] K. S. Rao, M. K. Reddy, J. L. Horning, V. Lahasetwar, Biomaterials 2008, 33, 4429 .

[22] X. M. Wang, N. Chi, X. Tang, Eur. J. Pharm. Biopharm. 2008, 70, 735.

[23] L. Costantino, F. Gandolfi, L. Bossy-Nobs, G. Tosi, R. Gurny, F. Rivasi, M. A. Vandelli, F. Forni, Biomaterials 2006, 27, 4635.

[24] J.-B. Kim, J. S. Choi, K. Nam, M. Lee, J.-S. Park, J.-K. Lee, J. Controlled Release 2006, 114, 110.

[25] L. Bracci, C. Falciani, B. Lelli, L. Lozzi, Y. Runci, A. Pini, M. G. De Montis, A. Tagliamonte, P. Neri, J. Biol. Chem. 2003, 278, 46590.

[26] G. Wu, R. F. Barth, W. Yang, S. Kawabata, L. Zhang, K. GreenChurch, Mol. Cancer Ther. 2006, 5, 52.

[27] C. C. Lee, J. A. MacKay, J. M. Fréchet, F. C. Szoka, Nat. Biotechnol. 2005, 23, 1517.

[28] E. R. Gillies, J. M. Fréchet, Drug Discov. Today 2005, $10,35$.

[29] N. Malik, R. Wiwattanapatapee, R. Klopsch, K. Lorenz, H. Frey, J. W. Weener, E. W. Meijer, W. Paulus, R. Duncan, J. Controlled Release. 2000, 65, 133.

[30] J. M. Oliveira, N. Kotobuki, A. P. Marques, P. Pirraco, J. Benesch, M. Hirose, S. A. Costa, J. F. Mano, H. Ohgushi, R. L. Reis, Adv. Funct. Mater. 2008, 18, 1840.

[31] J. Lu, Y. Wu, N. Sousa, O. F. Almeida, Development. 2005, 132, 3231.

[32] A. J. Salgado, R. A. Sousa, J. S. Fraga, J. M. Pego, B. A. Silva, J. O. Malva, N. M. Neves, R. L. Reis, N. Sousa, J. Bioact. Comp. Polym. 2009, 24, 235.

[33] A. H. Cory, T. C. Owen, J. A. Barltrop, J. G. Cory, Cancer Commun. 1991, 3, 207.

[34] V. Salih, K. Franks, M. James, G. W. Hastings, J. C. Knowles, J. Mater. Sci. Mater. Med. 2000, 11, 615. 